Research Article

\title{
A Simple and Scalable Method for the Preparation of Magnetite/Graphene Oxide Nanocomposites under Mild Conditions
}

\author{
Tamás Szabó ${ }^{(D)}{ }^{1}$ Lilla Nánai, ${ }^{1}$ Dániel Nesztor, ${ }^{1}$ Balázs Barna, ${ }^{1}$ Ondřej Malina, ${ }^{2}$ and \\ Etelka Tombácz ${ }^{1}$ \\ ${ }^{1}$ Department of Physical Chemistry and Materials Science, University of Szeged, Aradi vértanúk tere 1, Szeged 6720, Hungary \\ ${ }^{2}$ Regional Centre of Advanced Technologies and Materials, Department of Experimental Physics and Physical Chemistry, Faculty \\ of Science, Palacký University in Olomouc, Šlechtitelu 27, 78371 Olomouc, Czech Republic
}

Correspondence should be addressed to Tamás Szabó; sztamas@chem.u-szeged.hu

Received 5 September 2017; Accepted 31 October 2017; Published 21 January 2018

Academic Editor: Ilia Ivanov

Copyright (c) 2018 Tamás Szabó et al. This is an open access article distributed under the Creative Commons Attribution License, which permits unrestricted use, distribution, and reproduction in any medium, provided the original work is properly cited.

Nanostructured composite dispersions containing magnetic nanoparticles (MNPs) and graphene oxide (GO) lamellae have been prepared by a simple and easily scalable room temperature procedure. We show that, owing to the enormous surface area and negative surface charge developed in aqueous GO suspensions, large amounts of positively charged MNPs can be electrostatically attached to the layered host. This procedure is superior to many previous synthesis pathways because it exploits the chargeregulated adhesion of naked MNPs to GO resulting in the formation of stable and uniform nanocomposite materials in a wide composition range without any preliminary functionalization steps or harsh conditions that may lead to chemical degradation of the graphene-based nanosheets.

\section{Introduction}

Functional magnetic nanocomposites have been the focus of recent research due to their envisioned applications in biomedical, pharmaceutical, environmental, or nanoelectronic fields [1-5]. The most efficient use of these systems inevitably requires the precise control over their chemical and nanostructure. The elaboration of fast, efficient, and easily scalable methods is then pursued, which enables to obtain tailor-made synthesis solutions for specific applications. With the advent of graphene in emerging technologies, many studies have been published in the recent few years about the preparation and characterization of magnetically modified graphene-based materials. Graphene oxide (GO) is the most prominent host of magnetic nanoparticle (MNP) composites because its properties make it advantageous for large-scale production. Owing to their polar and ionisable surface sites [6], GO particles and their functionalized derivatives $[7,8]$ are easily processable in aqueous media, which favors the interaction of the lamellar host with the simplest, water-soluble iron salts (e.g., $\mathrm{FeSO}_{4}$ or $\mathrm{FeCl}_{3}$ ) and complexes as precursors [9-12]. Graphenebased composites are then obtained by transformation of $\mathrm{GO}$ to reduced graphene oxide (RGO). Extensive literature survey reveals three general synthesis routes for the formation of magnetic graphene particles: hydrothermal (more broadly and solvothermal) approaches [13-21], direct pyrolysis [9, 11, 22], and coprecipitation [23-30], all of which rely on in situ crystallization of MNPs on the carbonaceous layers and usually lead to the simultaneous reduction of GO to RGO.

However, some potential applications (e.g., drug delivery $[31,32])$ strongly demand that the oxidized form of the carbon counterpart of the magnetic composite remains unaltered after the composite formation. Although the coprecipitation $[33,34]$ and chemical grafting $[35,36]$ methods have been used under relatively mild conditions $\left(\sim 80^{\circ} \mathrm{C}\right.$ reaction temperature), the presence of bases at 
elevated temperatures inevitably results in partial deoxygenation of the GO framework [37, 38]. Another, more viable strategy is the preliminary functionalization of the magnetite nanoparticles [39], sulfonation of graphene oxide [40], or derivatization of both constituents by coupling azide-modified MNPs with alkyne-functionalized GO as reported by Namvari and Namazi [41]. The drawback of such routes is that they often require more tedious and complicated operations. Thus, it is still desirable to develop a more facile and efficient method for the preparation of magnetically modified GO materials.

In the present work, we report on a one-pot scalable method for the combination of magnetic nanoparticles with graphene oxide nanosheets exploiting the electrostatic interaction between their colloidal particles in aqueous solutions as provided by the development of their opposite surface charge. The structural and colloidal characteristics of the single and nanocomposite dispersions are characterized in detail. Up to now, to the best of our knowledge, there have been no other reports on the stable and strong attachment of $\mathrm{Fe}_{3} \mathrm{O}_{4}$ to graphene oxide in the absence of any functionalization scheme involved in the synthesis process.

\section{Materials and Methods}

2.1. Materials. For the synthesis of graphite oxide, we used purified (99.9 wt.\% C) graphite flakes of natural origin (codenamed as SGA-20) purchased from Kropfmühl $\mathrm{GmbH}$, Germany. The samples were also fractionated by the manufacturer $\left(d_{90}<50 \mu \mathrm{m}, d_{50}=16-21 \mu \mathrm{m}\right) . \mathrm{NaNO}_{3}$, $\mathrm{KMnO}_{4}$, the iron precursor salts, concentrated sulfuric acid, and 30 wt.\% hydrogen peroxide solutions were purchased from Molar Chemicals Ltd., Hungary. $\mathrm{NaCl}, \mathrm{NaOH}$, and $\mathrm{HCl}$ solutions used for maintaining constant background salt concentrations and constant $\mathrm{pHs}$ were used in analytical purity (Reanal). Water was deionised by ion exchange only for dialysis, while it was deionised by reverse osmosis and purified from particulate contaminants for all of the syntheses and light-scattering studies by Zeneer Power (TOC)RO\&UP System.

\subsection{Synthesis of Graphite Oxide by Hummers-Offeman} Method. A round-bottom flask was filled with $1 \mathrm{~g}$ of graphite and $1 \mathrm{~g}$ of $\mathrm{NaNO}_{3}$, which have been mixed together before $30 \mathrm{~mL}$ of $\mathrm{cc} . \mathrm{H}_{2} \mathrm{SO}_{4}$ was added. The suspension was stirred continuously with a magnetic stirrer, and $3 \times 1 \mathrm{~g}$ of $\mathrm{KMnO}_{4}$ was added in three portions hour by hour, upon which the thick slurry turned to green and became more viscous due to the swelling of the graphite structure and the formation of graphite bisulphate. It was then heated to $52 \pm$ $3^{\circ} \mathrm{C}$ and kept at this temperature for 6 hours. The oxidation reaction was terminated by the slow addition of $30 \mathrm{~mL}$ water close to $0^{\circ} \mathrm{C}$, and then $\mathrm{H}_{2} \mathrm{O}_{2}$ solution $(\sim 2 \mathrm{~mL})$ was gradually added until the suspension turned to golden brown and the effervescence discontinued. The as-obtained acidic slurry was centrifuged for 10 minutes at $3400 \mathrm{rpm}$ 3-4 times with intermittent removal of the supernatant and redispersion of the settled GO particles. During the successive increase of the $\mathrm{pH}$ of the dispersion medium, GO particles became colloidally stable, and centrifugation was incapable to remove them from the liquid phase. Therefore, the wet sediment was transferred to dialysis bags and dialyzed for ca. 10 days with periodic change of deionized water until its conductivity was steadily below $10 \mu \mathrm{S} / \mathrm{cm}$. GO was stored in aqueous suspension form, the concentration of which was determined by evaporating a known mass of dispersion to air-dry state.

2.3. Synthesis of MNPs. Magnetite nanoparticles were synthesized by alkaline hydrolysis [42-44] of $\mathrm{FeCl}_{2} \times 4 \mathrm{H}_{2} \mathrm{O}$ and $\mathrm{FeCl}_{3} \times 6 \mathrm{H}_{2} \mathrm{O}$. The concentrated solutions of iron (II) and iron (III) salts were mixed in the molar ratio of 1.1 to 2 and filtered into fresh water using a microfilter of $0.2 \mu \mathrm{m}$ pore size. The calculated amount of freshly prepared $\mathrm{NaOH}$ solution was added in $10 \%$ excess to the doubly diluted iron salt solution in two portions, the first half slowly and the second half rapidly under rigorous stirring. The formed black suspension was stirred for few minutes and then transferred into a larger amount of water. The suspension was washed with water several times to eliminate the alkaline impurities from synthesis, then acidified with $\mathrm{HCl}$ solution down to $\mathrm{pH} 2$, and washed again with water until disaggregation. The next step was the hydrothermal ageing of stable suspension in an ultrasonic bath at $80^{\circ} \mathrm{C}$ for 30 minutes. Finally, it was dialyzed against $0.001 \mathrm{M} \mathrm{HCl}$. The magnetite concentration was determined gravimetrically from a sample dried at $105^{\circ} \mathrm{C}$. The stock suspension $(5.78 \mathrm{~g}$ magnetite in $100 \mathrm{~g} 0.001 \mathrm{M}$ $\mathrm{HCl}$ ) was stored in the dark at $4^{\circ} \mathrm{C}$.

2.4. Preparation of GO/MNP Nanocomposites. $10 \mathrm{~g} / \mathrm{L}$ aqueous suspensions of the magnetic iron oxide and the graphite oxide phases directly diluted from the respective stock dispersions were used for the formation of composite materials. They were sonicated for $30 \mathrm{~s}$ before rapidly mixing them in appropriate volumes and, if necessary, adding water so that the total concentration of magnetite was $5 \mathrm{~g} / \mathrm{L}$. The concentration of GO was varied so that composite samples within the GO/MNP weight ratio range of $1 / 50$ to $10 / 1$ were obtained and stored in liquid-dispersed state for further studies. The final $\mathrm{pH}$ of suspensions were within the $\mathrm{pH}=4.5-6$ range.

2.5. Characterization. X-ray diffraction (XRD) analysis was performed by a Bruker D8 Advance diffractometer equipped with a Göbel mirror to yield a parallel beam of $\mathrm{CuK} \alpha$ radiation, which passed through a $1.2 \mathrm{~mm}$ slit. The X-ray tube was operated with $40 \mathrm{kV}$ acceleration voltage at $30 \mathrm{~mA}$ current. The diffraction data were collected with a count time of $1 \mathrm{~s}$ per step and sampling width of $0.02^{\circ}$.

FTIR measurements were conducted in attenuated total reflection (ATR) mode using a Bio-Rad Digilab Division FTS-65A/896 instrument equipped with a single reflection ATR accessory made of diamond and a DTGS detector. 128 scans were collected and averaged to obtain the spectra in $2 \mathrm{~cm}^{-1}$ digital resolution. 
Raman spectra were collected on a Thermo Scientific DXR Raman microscope equipped with a diode-pumped frequency-doubled Nd-YAG laser. Samples were irradiated at a wavelength of $532.2 \mathrm{~nm}$, with the laser beam focused using a $20 \times$ objective lens. $1 \mathrm{~mW}$ laser power was used to record the spectra. The instrument had a spectral resolution better than $2 \mathrm{~cm}^{-1}$ and a spatial resolution of a few cubic micrometers as provided by a $50 \mu \mathrm{m}$ slit confocal aperture.

TEM micrographs were taken by a JEOL JEM-1400+ transmission electron microscope operating at an accelerating voltage of $80 \mathrm{kV}$. One droplet of diluted nanocomposite dispersion was dried onto a carbon film-coated copper grid.

Dynamic light scattering measurements were taken by a NanoZS apparatus (Malvern) equipped with a $4 \mathrm{~mW}$ He-Ne laser source $(\lambda=633 \mathrm{~nm})$. The scattered light intensity was measured at an angle of $173^{\circ}$. The same instrument enabled for the measurement of the electrokinetic potential of the composite particles that were determined in the same dispersions as used for size measurements but using disposable zeta cells (DTS 1070) instead of plastic cuvettes. Composite samples were measured in highly diluted suspension to avoid significant interparticle interactions (hydrodynamic interaction or multiple light scattering).

A superconducting quantum interference device (SQUID) magnetometer (MPMS XL-7 type, Quantum Design, U.S.A.) was employed for the magnetization measurement at $300 \mathrm{~K}$. The hysteresis loop of the naked MNP sample was measured in an external magnetic field ranging from -50 to $+50 \mathrm{kOe}$. The magnetization values were corrected assuming the response of the sample holder and respective Pascal constants.

Magnetic fluid hyperthermia measurements were carried out by a magneTherm ${ }^{\mathrm{TM}}$ (Nanotherics, UK) system [45, 46]. The magnetic material content of each sample (bare MNP and nanocomposites at $1 / 50$ and $1 / 5$ mass ratios) was the same for all samples $(5 \mathrm{~g} / \mathrm{L})$. After ultrasonication of $1 \mathrm{~mL}$ of each sample for $20 \mathrm{~s}$, the heating efficiency was measured at a resonant frequency of $109.4 \mathrm{kHz}$ with magnetic field of $B=24.67 \mathrm{mT}(H=19.9 \mathrm{kA} / \mathrm{m}, 17$ turn coil, and $200 \mathrm{nF}$ capacitor) for $300 \mathrm{~s}$. SAR (specific absorption rate) values were not calculated from the initial slope of heating curves because their initial slopes were unusual for the nanocomposites and they differ from that of the naked MNP sample probably due to their aggregation.

\section{Results and Discussion}

3.1. Properties of Graphite Oxide. Figure 1 shows the XRD patterns of graphite before and after its harsh oxidation treatment. Graphite powder shows an outstandingly intense reflection centered at the diffraction angle of $26.6^{\circ}$. The respective c-axis repeat distance is $3.35 \AA$, which represents the spacing between graphene layers in well-crystallized graphite grains. This (002) reflection is shifted towards much lower angle values, the position of which was found to vary with ambient humidity conditions. The typical pattern of GO features two bands, one centered at $11^{\circ}$, while for the secondary one appearing with much lower intensity, the diffraction angle

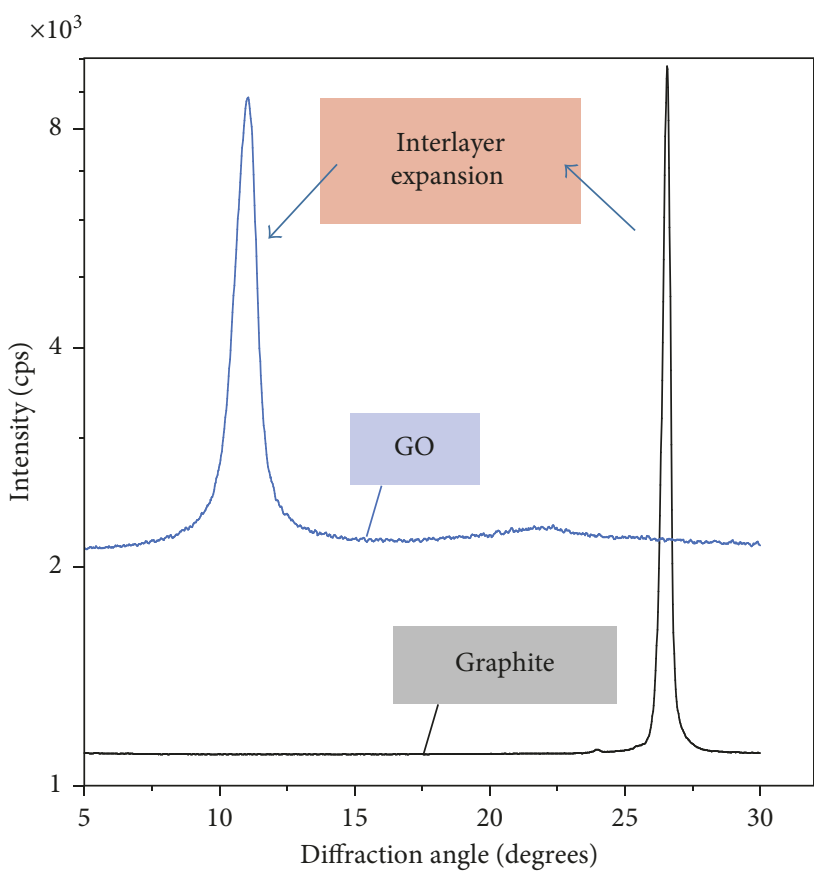

FIgURE 1: X-ray diffractograms of graphite and graphite oxide powders. For clarity, patterns are offset along the $y$-axis, and for graphite, it was divided by ten.

is doubled. The two peaks refer to the same periodic distance of $8.04 \AA$, indicating that the crystal structure of graphite expands to accommodate oxygenated functional groups and water molecules. Noteworthy is the broadening of the GO reflection as compared to graphite, which may be either caused by the nonuniform loading and distribution of water molecules between the graphene oxide sheets or by a decrease in the average flake size of graphite particles in the direction perpendicular to the carbon planes upon oxidation.

Raman spectroscopy is reported as a noninvasive tool to characterize the degree of oxidation and structure of graphene-based materials. The upper side spectrum of Figure 2 shows the Raman spectrum with well-resolved peaks characteristic for the graphite structure. The most intense and the sharpest one is the so-called G-band, which is the primary mode in many carbon materials representing the planar configuration of $\mathrm{sp}^{2}$ hybridized bonds that hold together the largest part of the atomic framework of the stacked graphene sheets. It appears at $1582 \mathrm{~cm}^{-1}$, which is identical to the respective wavenumbers of the G-bands for various graphite samples (involving highly oriented pyrolytic graphite) commonly reported in the literature [47-50]. Another in-plane vibration mode that also appears for the present and, in general, all previously studies of graphite samples is the $2 \mathrm{D}$ band at $2722 \mathrm{~cm}^{-1}$, which is a second-order overtone of the D-band centered at $1360 \mathrm{~cm}^{-1}$. The latter, first-order peak is not visible in perfect graphene owing to crystal symmetry considerations detailed in [47], in contrast to the parent graphite of our GO samples. The prerequisite of this band to appear is that defect or zone boundaries are abundant in the samples that enable a secondary elastic scattering of a phonon. Surprisingly, such defects are already 


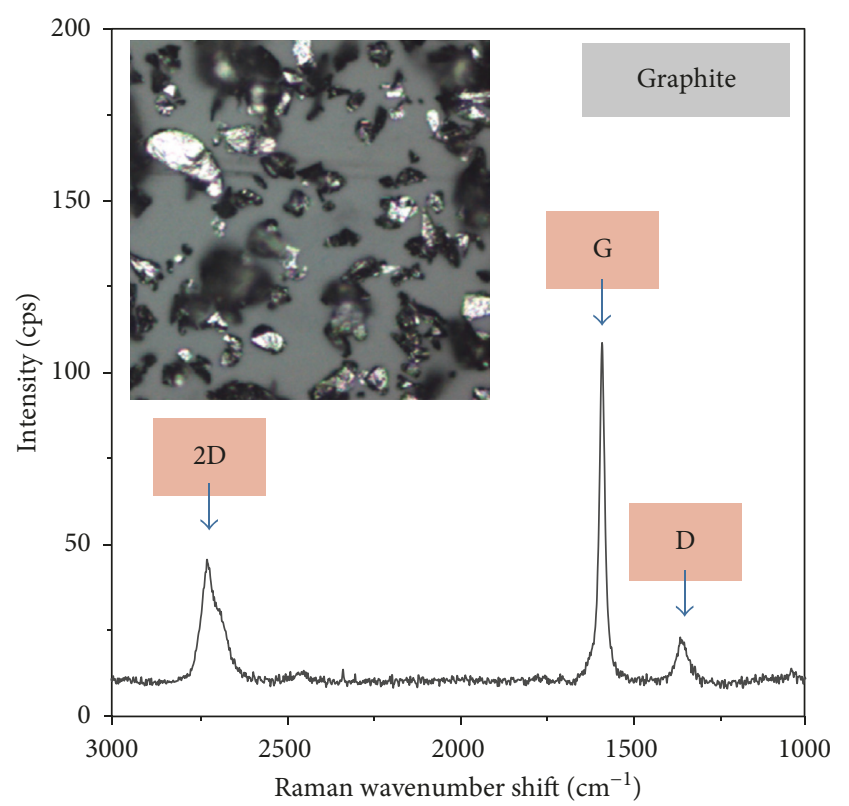

(a)

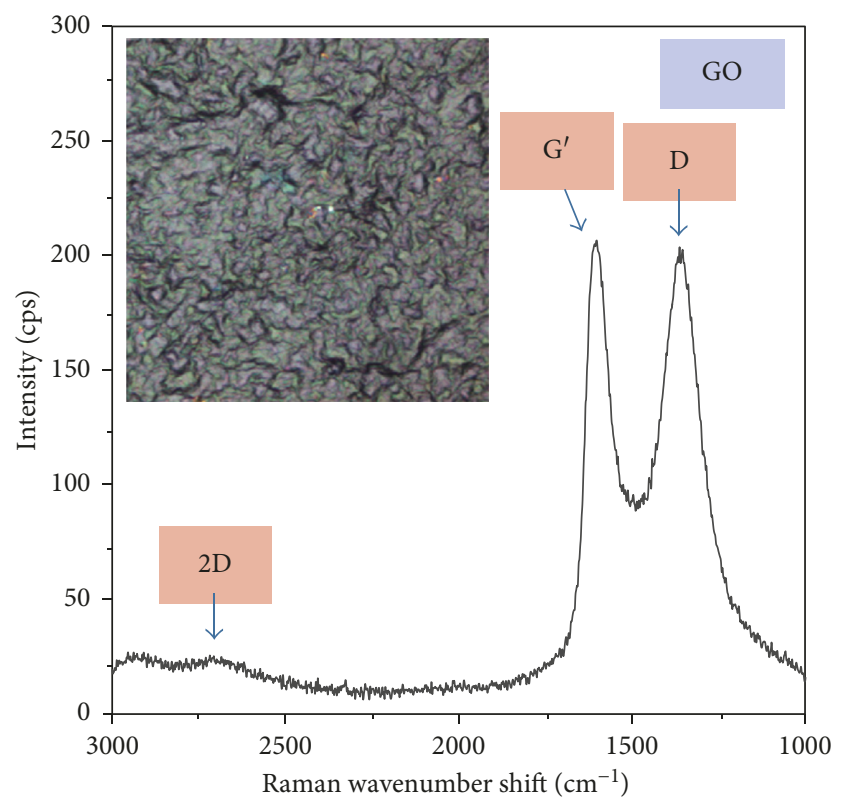

(b)

FIGURE 2: Raman spectra of graphite flakes (a) and graphite oxide powder (b). The insets show the optical microscopic image (at 50x magnification) of the samples.

present to a relatively high extent in the pristine graphite. Since the manufacturer does not report any harsh pretreatment of the present samples, we think that the grains are composed of very polydisperse multilayer stacks and the nanolamellar fraction with edges constructed of $\mathrm{sp}^{3}$ carbon atoms are present in relatively large proportion. The spectrum of graphite oxide (Figure 2(b)) proves that significant changes occurred by the oxidation of the carbon lattice. The most remarkable feature is the enhancement of disorderrelated bands. The $\mathrm{D}$-band shows height equal to the primary in-plane vibrational mode, while a scattering peak

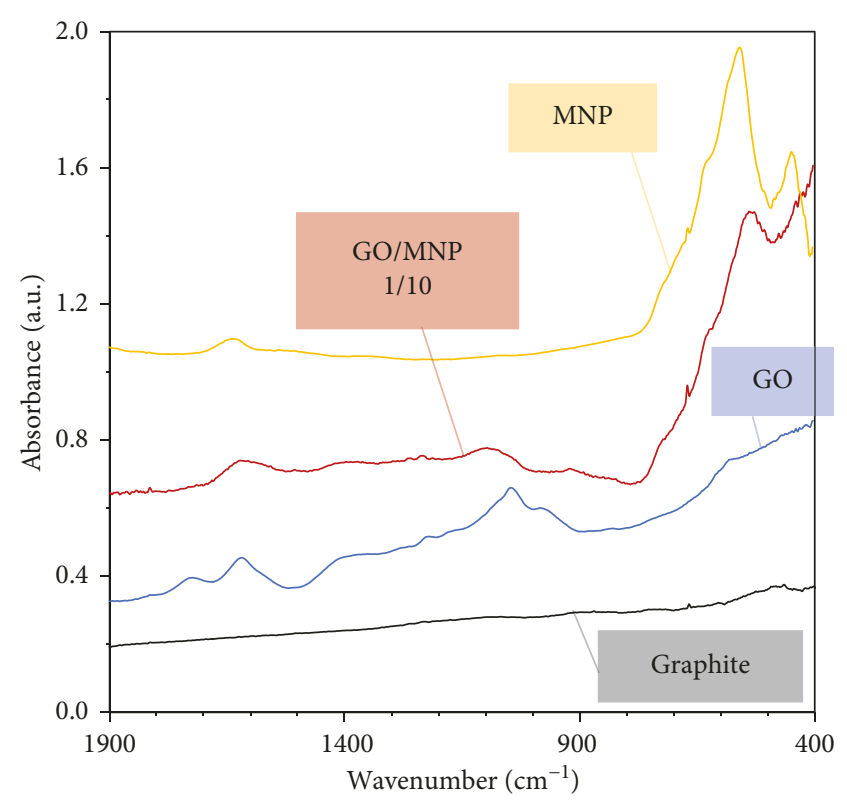

FIgURE 3: ATR-FTIR spectra of graphite, graphite oxide, bare MNP, and the GO/MNP nanocomposite at 1/10 mass ratio. The spectra are offset along the $y$-axis for clarity.

$\left(\nu=2954 \mathrm{~cm}^{-1}\right)$ appears at the higher wavenumber side of the $2 \mathrm{D}$ band which is ascribed to the combination of $\mathrm{D}+\mathrm{G}$ modes [51]. The last prominent feature for GO is that the G-band is blue shifted by $21 \mathrm{~cm}^{-1}$ to $1603 \mathrm{~cm}^{-1}$. This is originally present as a high-energy shoulder in graphite $\left(G^{\prime}\right)$, but for highly disordered carbons such as GO, it refers to an abundance of $\mathrm{sp}^{3}$ carbon atoms similar to that obtained upon physical (e.g., electron beam irradiation [52]) or chemical (strong oxidation) impacts. The inserted microscopic images in Figure 2 confirm the strong structural transformation: multilayered flakes of graphite are transformed into a puckered thin film state where the phase boundaries of individual, wrinkled particles are no longer observable.

Changes in the ATR-FTIR spectra brought by the oxidation reaction are indicated in Figure 3 . In the most informative spectral region, graphite exhibits a featureless spectrum indicating the absence of any functional groups. On the other hand, several bands are distinguishable in the spectrum of graphite oxide. The first one appears at $1734 \mathrm{~cm}^{-1}$, and it is commonly assigned to the presence of functional groups containing the $\mathrm{C}=\mathrm{O}$ motif. Carbonyl groups have been mostly suggested as part of the $\mathrm{COOH}$ moiety, while other assignments include single ketones or quinones [53]. At $1625 \mathrm{~cm}^{-1}$, the bending vibration of physically adsorbed water molecules gives rise to a peak. This is followed by a broad hump with several weak and poorly resolved bands emerging from the baseline. Only two of them can be associated with specific oxygenated functionalities: tertiary alcohol groups around $1400 \mathrm{~cm}^{-1}$ and phenolic $\mathrm{OH}$ around $1200 \mathrm{~cm}^{-1}$; however, their exact positions cannot be exactly assessed. Finally, the spectrum of GO represents several peaks at the lowest wavenumbers originating from $\mathrm{C}-\mathrm{C}$ and $\mathrm{C}-\mathrm{O}$ lattice vibration modes. 


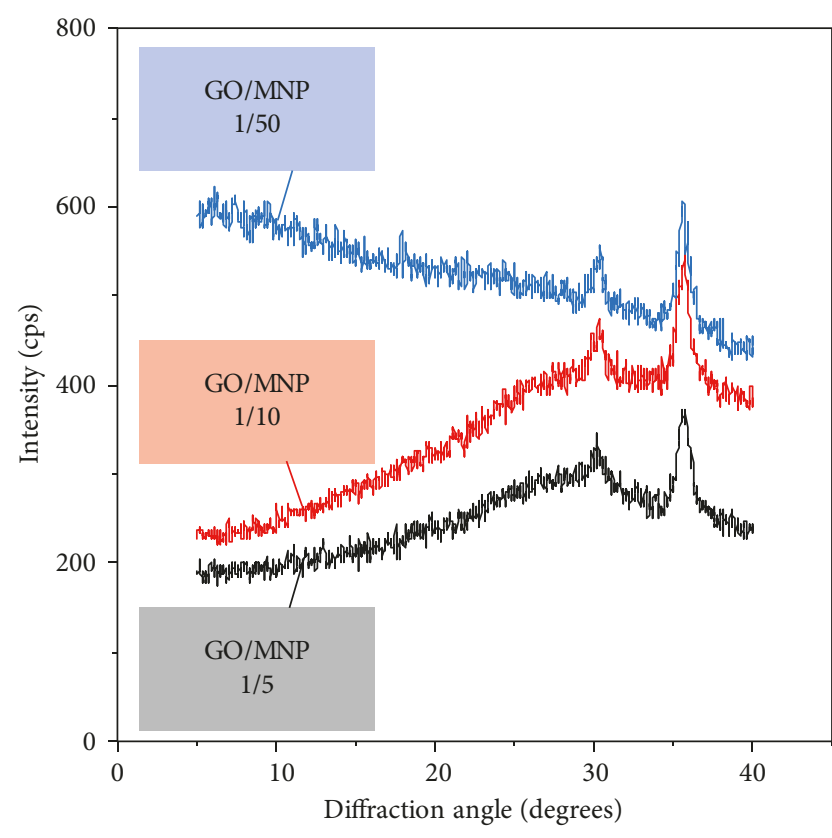

FIGURE 4: XRD patterns of GO/MNP nanocomposites at different mass ratios. For clarity, patterns are offset by $100 \mathrm{cps}$.

3.2. Structural Properties of GO/MNP Nanocomposites. Figure 4 shows the X-ray diffractograms of the magnetite nanocomposites loaded with different amounts of GO. Two reflections are distinguished in these samples (at $2 \Theta=30.36^{\circ}$ and $35.6^{\circ}$ ) that belong to the (220) and (311) lattice planes of the cubic magnetite crystal structure [54]. Since the dominant mass in all samples belongs to the MNP phase, the respective peak intensities do not show much variation with the composition. An average crystallite diameter was calculated from the broadening of the (311) reflections, and they were found to be $10 \mathrm{~nm}$ and obviously did not change during composite formation. Although the apparent breadth of the (220) reflection is larger for the GO/MNP $1 / 5$ composite, this is solely due to the irregular baseline that originates from the fluorescence of the $\mathrm{CuK} \alpha$ radiation on iron centers. The most interesting feature of the powder X-ray patterns is the complete disappearance of the reflection at low angles that is characteristic for the interplanar distance of graphite oxide particles. This clearly shows that the carbon-based phase is dispersed to the level of individual graphene oxide particles. It is known that high degrees of exfoliation are achieved in aqueous GO suspensions since many atomic force microscopic studies imaged $1 \mathrm{~nm}$ high platelets deposited from the liquid phase [55]. During composite formation with the magnetic phase, the liquid-suspended GO nanosheets exhibit an enormously high specific surface area available for the attachment of the MNPs. When the dispersion medium is dried off from the composite particles, the coated lamellae must be restacked and become rotationally disordered but parallelly aligned to the sample holder, capturing iron oxide particles between the layers. Obviously, the repeat distances in the expanded layer structure are then too large to give any diffraction peak in the studied angle range. However, it might be somewhat surprising that the GO peak is absent even at the highest GO/MNP ratios of $1 / 5$, for which the number of sheets may exceed the necessary area needed to accommodate the magnetite nanoparticles, and restacked empty areas could still give rise to an interlayer distance similar to that found for bare GO. This phenomenon implies that the composite particles may retain a "house of cards" structure in the dried state with single- or few-layered GO particles present in the matrix of the aggregated mass of the magnetite nanoparticles.

The ATR-FTIR spectrum of the nanocomposite of GO/MNP ratio of $1 / 10$ is compared to that of the bare magnetite phase in Figure 3. The latter shows two peaks, one at $1620 \mathrm{~cm}^{-1}$ and another at $556 \mathrm{~cm}^{-1}$ with two minor shoulders appearing at around 630 and $700 \mathrm{~cm}^{-1}$. The former is due to the bending vibration of adsorbed water, and the others are attributed to different vibration modes of the Fe-O structure [56]. The nanocomposite shows very weak absorption at wavenumbers characteristic for GO, and the iron oxide-related peaks dominate the spectrum. However, the most intense band shows a red shift by $23 \mathrm{~cm}^{-1}$, indicating a slight weakening of the bond strength between $\mathrm{Fe}$ and $\mathrm{O}$ atoms. This might be caused by the extensive hydrogen bond network between the hydroxylated $\mathrm{GO}$ and $\mathrm{Fe}_{3} \mathrm{O}_{4}$ surfaces, but it is also possible that there is an abundance of positively charged surface sites $\left(\equiv \mathrm{Fe}-\mathrm{OH}_{2}^{+}\right)$developed on the iron oxide when it is contacted with GO.

Electron microscopy revealed important differences in the nanostructure of the GO/MNP composites at different compositions (Figure 5). At the lowest MNP loadings (GO/MNP mass ratio of $1 / 5$ ), the majority of the primary magnetic nanocrystals are well-distinguishable and clustering only occurs on a few locations of the GO surface, for example, near the top right corner of the image. With a few exceptions, the diameters of the particles fall into the range of 3-20 nm, which is typical for $\mathrm{Fe}_{3} \mathrm{O}_{4}$ nanophases exhibiting superparamagnetic behavior. The average size was $9.5 \mathrm{~nm}$, showing good agreement with the one determined by XRD line broadening. Graphene oxide is also visualized on the micrographs at relatively high magnifications. In the current image and in others taken at different areas of the sample, they appear as slightly curved lines with approximate length of $50 \mathrm{~nm}$, reminiscent of folded paper-like layers. These features give conclusive proof that the flexible particles are present in a highly delaminated, single-layer state, that otherwise do not give enough contrast for direct observation by TEM. Interestingly, a relatively large fraction of magnetite nanocrystals are attached to the graphene oxide platelets following the shape of these folds or along the platelet edges, suggesting that these surface features exert stronger adhesive forces towards the MNPs. Upon increasing the MNP loading to tenfold excess relative to GO (Figure 5(b)), the surface coverage of particles increased proportionally. The planar distribution of particles did not change with loading: sites densely covered by magnetite were loosely interconnected, leaving behind irregularly shaped patches over the uncoated graphene oxide surfaces of several hundreds to thousands of square nanometer surface area. However, at the highest GO/MNP ratio (1/50), the particles 


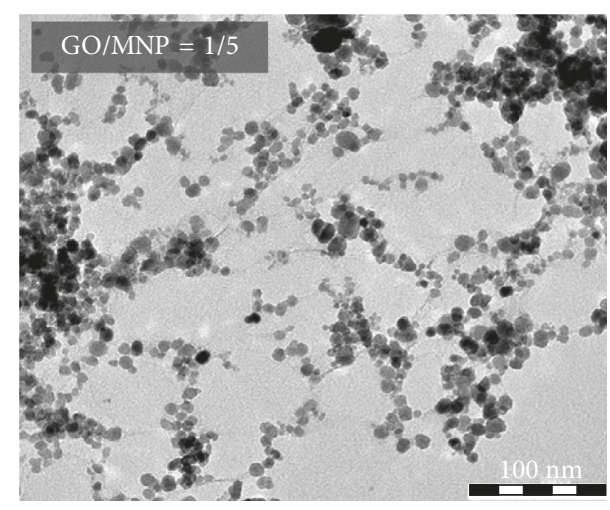

(a)

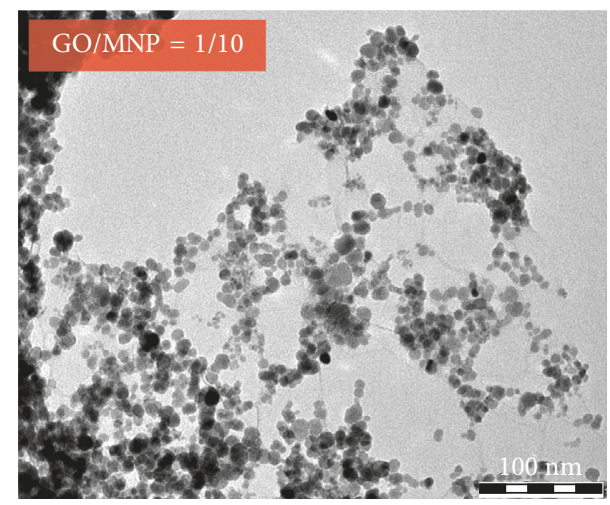

(b)

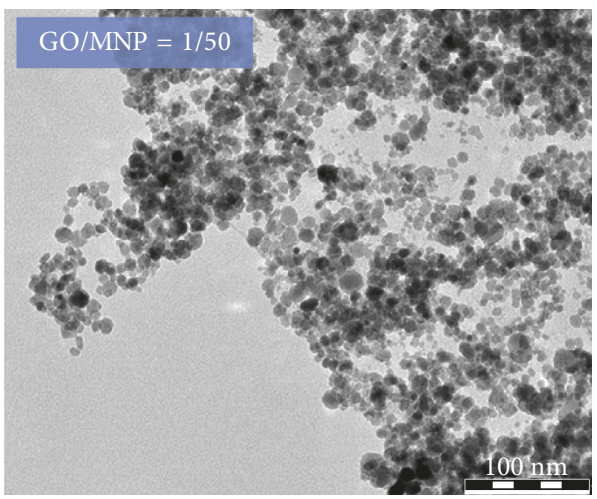

(c)

Figure 5: Electron micrographs of magnetic nanocomposites at different GO/MNP mass ratios of (a) 1 to 5 , (b)1 to 10 , and (c) 1 to 50 .

are almost fully covered, and the magnetic nanocrystals undergo significant clustering that is virtually extended to the whole imaged area.

Due to the high MNP contents, significant variations might occur at the microscopic level in the particle-toparticle compositions. Besides, once the available surface area becomes lower than that required for the deposition of the total amount of particles, the sample would lose phase homogeneity and become a mixture of a nanocomposite phase consisting of GO layers fully covered with the MNPs and a pure magnetite phase involving the rest of the MNPs. To check this possibility, TEM images were taken on the highly loaded samples at low magnifications, which were qualitatively similar to that presented in Figure 6. We

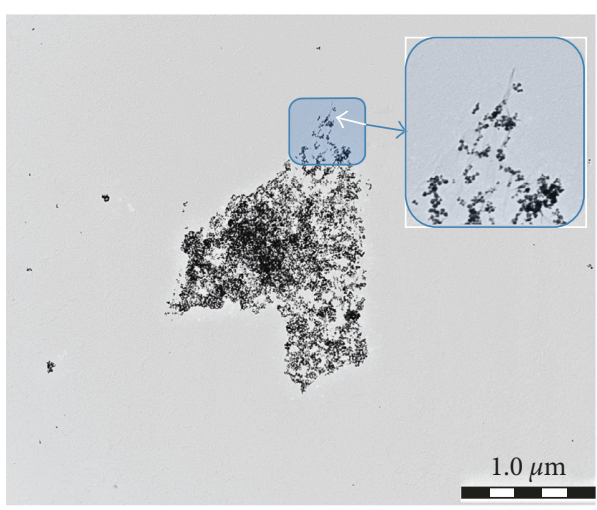

FIGURE 6: Electron micrograph of $1 / 10$ mass ratio GO/MNP composite at low magnification. The inset shows the enlarged edge region of the imaged particle. The scale bar refers to $1 \mu \mathrm{m}$ length.

observed that virtually all magnetic iron oxide particles were assembled only on the lamellar surfaces. This is another important finding because it indicates not only that MNPs are only dispersed on GO in the dry state but also that a stable nanocomposite phase was already present in the aqueous suspension. This latter statement is well established since it is highly unlikely that such a large amount of separated single or clustered nanocrystallites would have exclusively assembled on the GO platelet only because of the loss of the solvent.

3.3. Characteristics of Magnetic Nanocomposite Dispersions. The visual appearance of undisturbed aqueous GO/MNP dispersions of different compositions provides important indirect evidence on the interparticle interactions and the colloidal stability of the system. As seen from the photograph of Figure 7(a), freshly homogenized suspensions undergo rapid coagulation and start to settle immediately forming large floc-like secondary particles when their GO/MNP ratios range between 1 and 0.1. Aggregation also occurs at different loadings, as indicated by the sharp settling front in GO-rich samples, while the settling rate gradually increases with the magnetite loading (Figure 7(b)). However, when MNPs are in large excess, sedimentation slows down and becomes more diffuse, with a considerable fraction of particles remaining in suspended form after 1 hour ageing. Nevertheless, within one day, the entire amount of nanocomposite particles settles down independent of their composition (Figure $7(\mathrm{c})$ ). This is in sharp contrast with the settling behavior of the respective single-component particles (not shown), which retained their homogeneous spatial distribution in water for extended time periods. This macroscopic-scale observation is in excellent agreement with the one obtained by electron microscopy on the exclusive occurrence of MNP particles on the GO host lamellae: both of them suggest that there is a strong interaction between the $\mathrm{Fe}_{3} \mathrm{O}_{4}$ and the $\mathrm{GO}$ surfaces. One plausible explanation for the origin of the strong adhesive forces between these dissimilar colloidal particles is an extensive hydrogen bonding between the hydroxyl group containing surfaces of GO and MNP (for the former, they are originally 


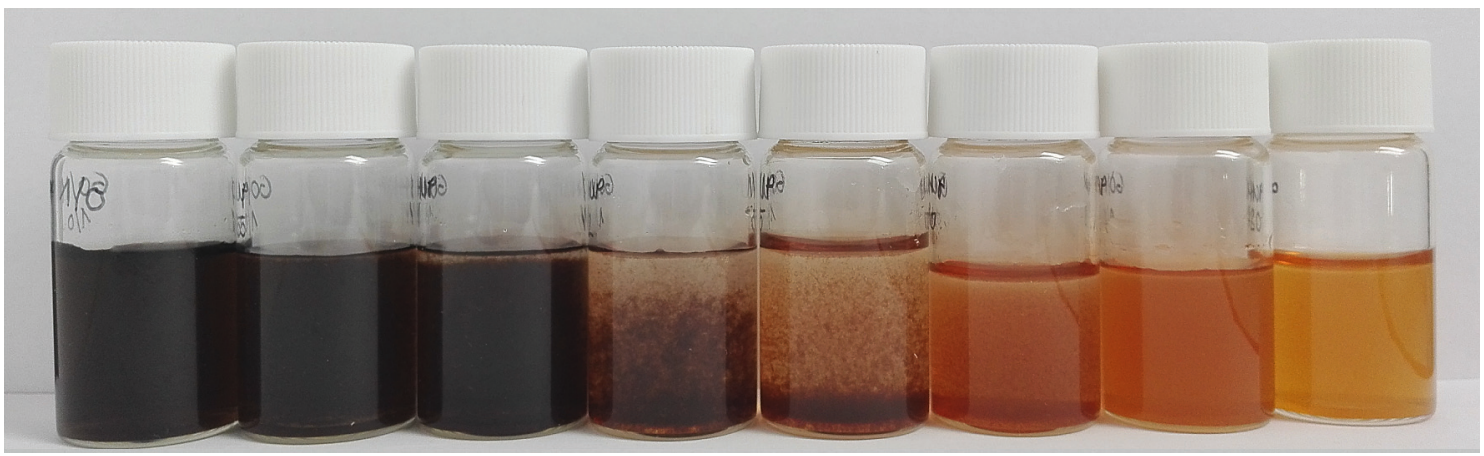

(a)

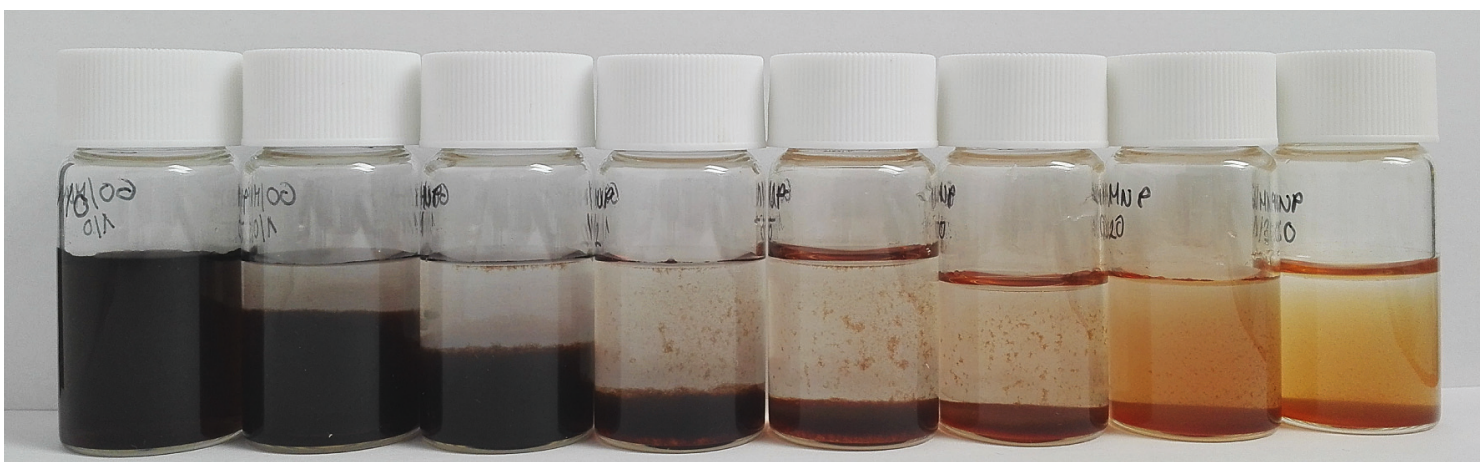

(b)

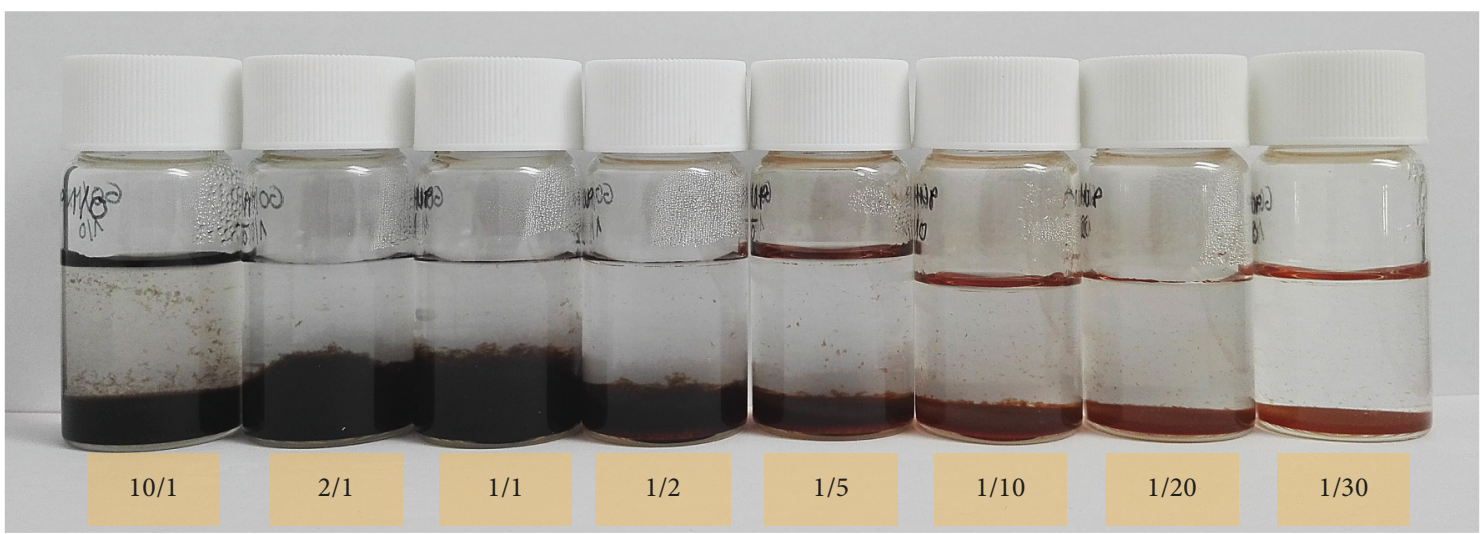

(c)

Figure 7: Photographs of settling nanocomposite dispersions immediately after homogenization (a), after one hour (b), and after one day (c). Numerical ratios indicated below (c) refer to different GO/MNP mass ratios.

part of the chemical structure, and for the latter, an abundance of $\mathrm{Fe}-\mathrm{OH}$ surface sites form upon dissociative chemisorption of water molecules [57]). However, it is more likely that strong coulombic attraction forces arise when the two different colloidal particles are mixed together in water. To check the validity of this possibility, electrokinetic measurements were performed as detailed later.

Turning back to the dispersion characteristics, the largest equilibrium sediment volume appears at the highest GO loadings, which is partly due to the fact that the total solid content in these suspension is larger (the mass concentration of MNP is always $5 \mathrm{~g} / \mathrm{L}$ in the whole series, and the GO excess mass was specified to this value). However, it is also possible that GO lamellae constitute a "house of cards" arrangement opening up the nanostructure, and the loose aggregates form a more voluminous sediment than those which are composed mostly of magnetite.

As a final note here, one would expect black or very dark brown color for all dispersions. However, when the magnetic particles become dominant in the samples, they turn to orange especially at higher dilutions. This is simply because of slow atmospheric oxidation of $\mathrm{Fe}_{3} \mathrm{O}_{4}$ to maghemite, but since this polymorph of iron oxide exhibits nearly the same magnetism and chemical properties [42], this transformation does not change crucially the characteristics of the GO/MNP composites.

Figure 8 shows the electrokinetic potentials and particle diameters (scattering intensity-based "Z-average") as the function of the composition. It is important to note that, in contrast to the general GO/MNP representation, it is 


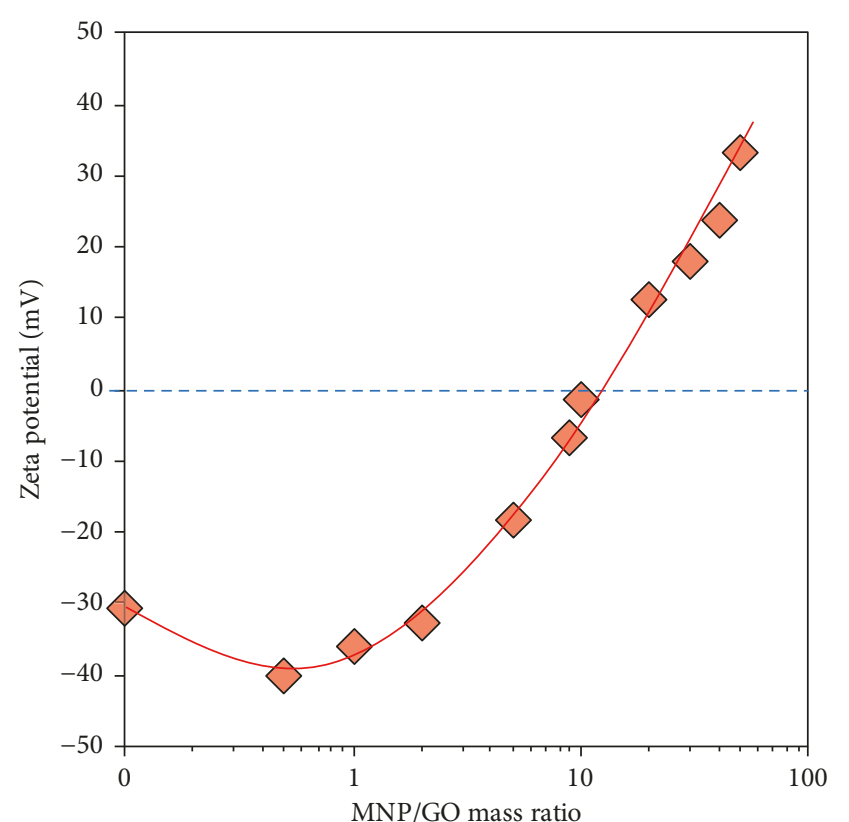

(a)

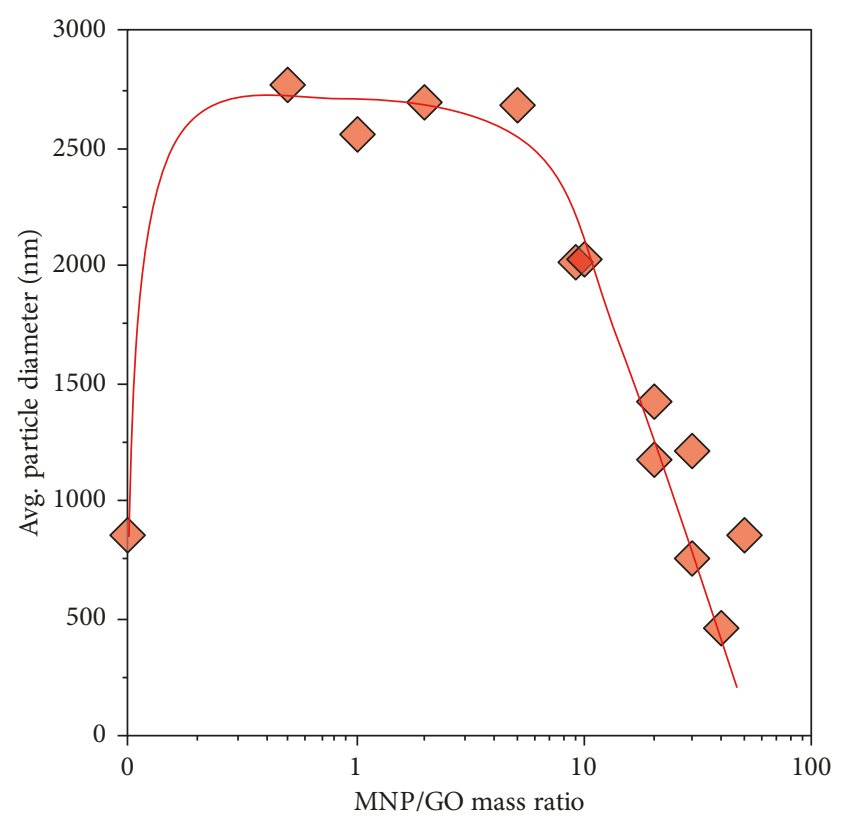

(b)

FIGURE 8: Electrokinetic potentials (a) and Z-average particle diameters (b) of the MNP/GO nanocomposite aqueous dispersions as a function of the MNP/GO mass ratio. Measurements refer to the $\mathrm{pH}$ range of 4.5-6.

expressed as the inverse mass ratio $(\mathrm{MNP} / \mathrm{GO})$ in order to be more conveniently comparable with the photographs in Figure 7. Thus, nanocomposite dispersion samples rich in the carbonaceous phase are on the left, while magnetite-rich samples are found on the right hand side of the photographs and the electrokinetic potencial-composition functions of Figure 8. The exfoliated graphite oxide particles are dispersed to the submicrometer level $\left(d_{\text {ave }}=800 \mathrm{~nm}\right)$, and their $\zeta$ potential is $-30 \mathrm{mV}$. The latter is the electric potential difference between the dispersion medium and the solvent

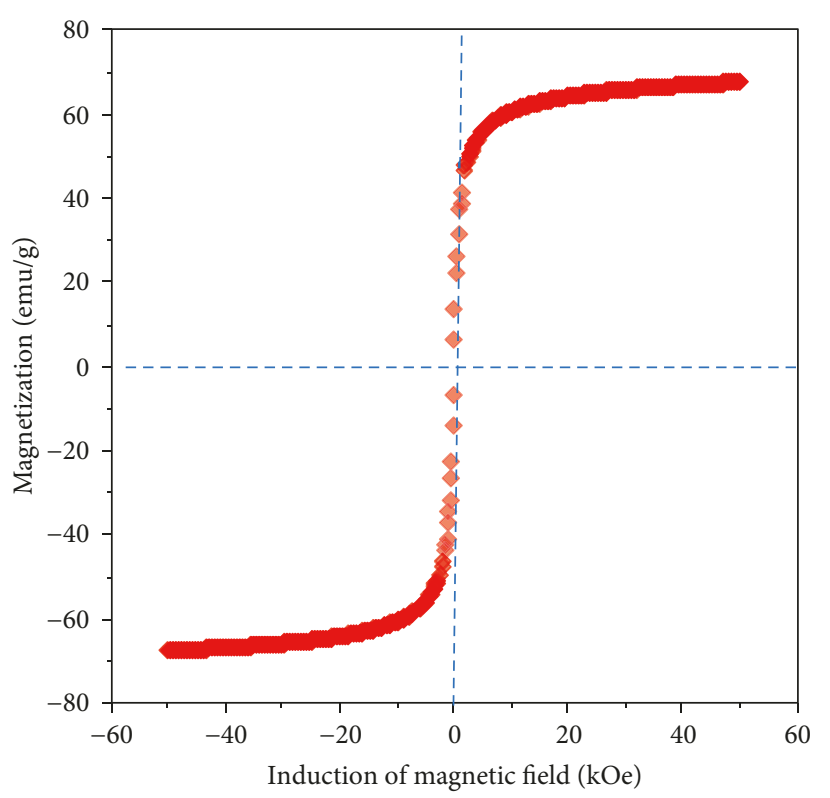

(a)

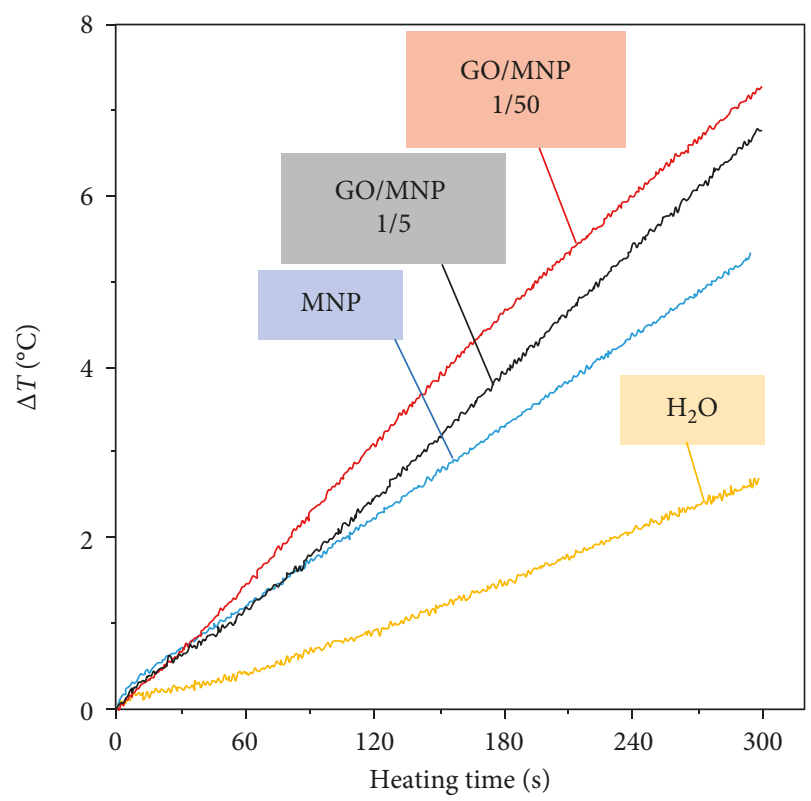

(b)

FIGURE 9: Magnetization curve of the MNPs (a) and heating curves (b) of nanocomposite dispersions of $5 \mathrm{~g} / \mathrm{L} \mathrm{MNP}$ content at two different GO/MNP ratios as compared to those of pure water and the bare MNP suspension of the same concentration.

layer adhered to the suspended solid particle upon its electrophoretic migration [58]. This potential is usually lower than that measured at the particle surface, but its sign and magnitude are a good indication of the surface charge state and kinetic stability of the dispersed material. It is a common observation that electrostatically stabilized colloidal systems aggregate when the $\zeta$ potential falls within the range of $\pm 20 \mathrm{mV}$. Graphene oxide particles show $\zeta$ potential far lower than this value, indicating that their surfaces are highly charged in water, in agreement with previous observations [59]. On the other hand, $+40 \mathrm{mV}$ electrokinetic potential is 
measured for naked magnetite nanoparticle sols at $\mathrm{pH}$ values below $\sim 5$ [44]. For the binary composites, the charge increases slightly at the highest excesses of GO probably due to slight $\mathrm{pH}$ variation, but then it starts to increase gradually with the addition of magnetic nanoparticles. This suggests that MNPs are adsorbed to the surface of graphene oxide lamellae by electrostatic attraction upon which they progressively screen the surface charge and, concomitantly, decrease the magnitude of $\zeta$ potential. The interaction of oppositely charged GO and magnetite nanoparticles results in aggregation. Huge secondary particles form in the lower MNP/GO mass ratio region (Figure $8(\mathrm{~b})$ ) probably due to the bridging of $\mathrm{GO}$ lamellae via MNPs. This finding is in good harmony with the visual observation of voluminous sediments formed mainly at 2/1 and 1/1 GO/MNP ratios after 1 day standstill (Figure 7 (c)), typical for house-of-cards arrangement of aggregates formed from anisometric, two-dimensional particles. However, as the MNP/GO mass ratio further increases, the average hydrodynamic diameters of dispersed particles successively decrease reaching the submicron scale. This implies, in good agreement with the magnitude of the particle dimensions of the single composite particle shown in Figure 6, that the degree of aggregation decreases with MNP loading. Eventually, primary GO sheets densely coated by MNPs dominate in the dispersed phase.

\subsection{Performance of $M N P / G O$ Composites in Magnetic} Hyperthermia. The potential use of the magnetic nanocomposites is demonstrated by their heating efficiency for a potential future use in hyperthermic cancer therapy [60]. Hyperthermia treatment relies on the temperature rise in a medium induced by the Néel and Brownian relaxation of superparamagnetic nanoparticles [61, 62]. The magnetization curve of the MNPs (Figure 9(a)) clearly proves that the iron oxide nanoparticles used in this study are in the superparamagnetic state exhibiting a saturation magnetization of $67.6 \mathrm{emu} / \mathrm{g}$. In Figure 9(b), the heating curves of GO/MNP composites at two different compositions are compared to the heating curve obtained for a magnetite suspension of the same solid content in the absence of carbonaceous lamellae. It was found that both nanocomposites generated larger heat effects than the suspension containing the magnetic nanoparticles as indicated by the relatively large difference in the temperature measured between the suspensions after 5 minutes of AC magnetic field exposure $\left(7.29^{\circ} \mathrm{C}\right.$ for $\mathrm{GO} / \mathrm{MNP}=1 / 5,5.27^{\circ} \mathrm{C}$ for pristine magnetite). This enhancement in the heating efficiency is even more remarkable ( $>75 \%$ for the $1 / 50$ composite) if one takes into account that pure water also produces heat under the same experimental conditions, caused by eddy currents [61]. We attribute this additional heat production to a resistance heating effect caused by Joule-losses of the graphene oxide particles. To our surprise, the hyperthermic effect was found to be larger at low GO loadings. Clearly, it is worth conducting further investigations, for example, on the composition dependence of the heating efficiency for these nanocomposite materials to tune their functionalities for specific applications.

\section{Conclusions}

The present study demonstrates that uniform GO/MNP nanocomposites can be easily formulated in a wide composition range utilizing the strong electrostatic interaction between their particles. Graphene oxide particles were able to accommodate MNPs that represent masses up to 50 times larger than the lamellar carbonaceous host. This development is significant especially because strong binding can be maintained between naked MNPs and GO particles without chemical coupling, which would require preliminary efforts for functionalization of both MNPs and GO [15, 36, 63, 64]. Moreover, the present synthesis does not require harsh chemical treatments or elevated temperatures, thereby keeping the chemical structure of graphene oxide intact. This is a definite benefit compared to recent studies $[65,66]$ reporting on the preparation of $\mathrm{MNP} / \mathrm{GO}$ composites by hydrothermal methods in which the temperature was maintained at $190^{\circ} \mathrm{C}$, where $\mathrm{GO}$ is expected to undergo significant thermal decomposition [67]. Finally, we showed that the method is versatile, enabling a large variation in the compositions affording highly different nanostructures ranging from "house of cards" arrangement of MNPdecorated exfoliated graphite oxide particles to GO nanosheets matrixed into bulk amounts of magnetite.

\section{Conflicts of Interest}

The authors declare that they have no conflicts of interest regarding the publication of this paper.

\section{Acknowledgments}

This work was financially supported by the Hungarian National Research, Development and Innovation Office via the Grant FK-124851.

\section{References}

[1] S. Behrens, "Preparation of functional magnetic nanocomposites and hybrid materials: recent progress and future directions," Nanoscale, vol. 3, no. 3, pp. 877-892, 2011.

[2] N. D. Q. Chau, C. Ménard-Moyon, K. Kostarelos, and A. Bianco, "Multifunctional carbon nanomaterial hybrids for magnetic manipulation and targeting," Biochemical and Biophysical Research Communications, vol. 468, no. 3, pp. 454-462, 2015.

[3] A. B. Bourlinos, M. A. Karakassides, A. Simopoulos, and D. Petridis, "Synthesis and characterization of magnetically modified clay composites," Chemistry of Materials, vol. 12, no. 9, pp. 2640-2645, 2000.

[4] V. Tzitzios, G. Basina, A. Bakandritsos et al., "Immobilization of magnetic iron oxide nanoparticles on laponite discs - an easy way to biocompatible ferrofluids and ferrogels," Journal of Materials Chemistry, vol. 20, no. 26, pp. 5418-5428, 2010.

[5] T. Szabó, A. Bakandritsos, V. Tzitzios et al., "Magnetic iron oxide/clay composites: effect of the layer silicate support on the microstructure and phase formation of magnetic 
nanoparticles," Nanotechnology, vol. 18, no. 28, Article ID 285602, 2007.

[6] T. Szabó, E. Tombácz, E. Illés et al., "Enhanced acidity and $\mathrm{pH}$-dependent surface charge characterization of successively oxidized graphite oxides," Carbon, vol. 44, no. 3, pp. 537-545, 2006.

[7] V. Georgakilas, M. Otyepka, A. B. Bourlinos et al., "Functionalization of graphene: covalent and non-covalent approaches, derivatives and applications," Chemical Reviews, vol. 112, no. 11, pp. 6156-6214, 2012.

[8] A. Bakandritsos, M. Pykal, P. Błoński et al., "Cyanographene and graphene acid: emerging derivatives enabling high-yield and selective functionalization of graphene," ACS Nano, vol. 11, no. 3, pp. 2982-2991, 2017.

[9] T. Szabó, A. Bakandritsos, V. Tzitzios et al., "Magnetically modified single and turbostratic stacked graphenes from tris (2, 2'-bipyridyl) iron (II) ion-exchanged graphite oxide," Journal of Physical Chemistry B, vol. 112, no. 46, pp. 1446114469, 2008.

[10] S. Bashkova and T. J. Bandosz, "Adsorption/reduction of $\mathrm{NO}_{2}$ on graphite oxide/iron composites," Industrial \& Engineering Chemistry Research, vol. 48, no. 24, pp. 1088410891, 2009.

[11] J. Shen, Y. Hu, M. Shi, N. Li, H. Ma, and M. Ye, "One step synthesis of graphene oxide-magnetic nanoparticle composite," Journal of Physical Chemistry C, vol. 114, no. 3, pp. 1498-1503, 2010.

[12] J. A. Arcibar-Orozco, D. A. Giannakoudakis, and T. J. Bandosz, "Effect of Ag containing (nano)particles on reactive adsorption of mustard gas surrogate on iron oxyhydroxide/graphite oxide composites under visible light irradiation," Chemical Engineering Journal, vol. 303, pp. 123-136, 2016.

[13] H.-P. Cong, J.-J. He, Y. Lu, and S.-H. Yu, "Water-soluble magnetic-functionalized reduced graphene oxide sheets: in situ synthesis and magnetic resonance imaging applications," Small, vol. 6, no. 2, pp. 169-173, 2010.

[14] K. Zhou, Y. Zhu, X. Yang, and C. Li, "One-pot preparation of graphene/ $\mathrm{Fe}_{3} \mathrm{O}_{4}$ composites by a solvothermal reaction," New Journal of Chemistry, vol. 34, no. 12, pp. 2950-2955, 2010.

[15] Y. Li, J. Chu, J. Qi, and X. Li, “An easy and novel approach for the decoration of graphene oxide by $\mathrm{Fe}_{3} \mathrm{O}_{4}$ nanoparticles," Applied Surface Science, vol. 257, no. 14, pp. 6059-6062, 2011.

[16] L. Ai, C. Zhang, and Z. Chen, "Removal of methylene blue from aqueous solution by a solvothermal-synthesized graphene/magnetite composite," Journal of Hazardous Materials, vol. 192, no. 3, pp. 1515-1524, 2011.

[17] J. Shen, M. Shi, H. Ma, B. Yan, N. Li, and M. Ye, "Hydrothermal synthesis of magnetic reduced graphene oxide sheets," Materials Research Bulletin, vol. 46, no. 11, pp. 2077-2083, 2011.

[18] Y.-W. Liu, M.-X. Guan, L. Feng et al., "Facile and straightforward synthesis of superparamagnetic reduced graphene oxide- $\mathrm{Fe}_{3} \mathrm{O}_{4}$ hybrid composite by a solvothermal reaction," Nanotechnology, vol. 24, no. 2, Article ID 025604, 2013.

[19] Y. Wang, Q. He, H. Qu et al., "Magnetic graphene oxide nanocomposites: nanoparticles growth mechanism and property analysis," Journal of Materials Chemistry C, vol. 2, no. 44, pp. 9478-9488, 2014.

[20] J. Qian, X. Yang, L. Jiang, C. Zhu, H. Mao, and K. Wang, "Facile preparation of $\mathrm{Fe}_{3} \mathrm{O}_{4}$ nanospheres/reduced graphene oxide nanocomposites with high peroxidase-like activity for sensitive and selective colorimetric detection of acetylcholine," Sensors and Actuators B, vol. 201, pp. 160-166, 2014.

[21] K. Luo, Y. Mu, P. Wang, and X. Liu, "Effect of oxidation degree on the synthesis and adsorption property of magnetite/graphene nanocomposites," Applied Surface Science, vol. 359, pp. 188-195, 2015.

[22] J. Zhou, H. Song, L. Ma, and X. Chen, "Magnetite/graphene nanosheet composites: interfacial interaction and its impact on the durable high-rate performance in lithium-ion batteries," RSC Advances, vol. 1, no. 5, pp. 782-791, 2011.

[23] Y.-X. Ma, Y.-F. Li, G.-H. Zhao et al., "Preparation and characterization of graphite nanosheets decorated with $\mathrm{Fe}_{3} \mathrm{O}_{4}$ nanoparticles used in the immobilization of glucoamylase," Carbon, vol. 50, no. 8, pp. 2976-2986, 2012.

[24] G. He, W. Liu, X. Sun, Q. Chen, X. Wang, and H. Chen, " $\mathrm{Fe}_{3} \mathrm{O}_{4} @$ graphene oxide composite: a magnetically separable and efficient catalyst for the reduction of nitroarenes," $M a$ terials Research Bulletin, vol. 48, pp. 1885-1890, 2013.

[25] Y. Zhao, J. Li, S. Zhang, H. Chen, and D. Shao, "Efficient enrichment of uranium(VI) on amidoximated magnetite/graphene oxide composites," RSC Advances, vol. 3, no. 41, pp. 18952-18959, 2013.

[26] M. Zong, Y. Huang, Y. Zhao et al., "Facile preparation, high microwave absorption and microwave absorbing mechanism of RGO- $\mathrm{Fe}_{3} \mathrm{O}_{4}$ composites," RSC Advances, vol. 3, no. 45, pp. 23638-23648, 2013.

[27] X. Hu, Y. Liu, H. Wang et al., "Removal of $\mathrm{Cu}$ (II) ions from aqueous solution using sulfonated magnetic graphene oxide composite," Separation and Purification Technology, vol. 108, pp. 189-195, 2013.

[28] V. Chandra, J. Park, Y. Chun, J. W. Lee, I.-C. Hwang, and K. S. Kim, "Water-dispersible magnetite-reduced graphene oxide composites for arsenic removal," ACS Nano, vol. 4, no. 7, pp. 3979-3986, 2010.

[29] M. Liu, C. Chen, J. Hu, X. Wu, and X. Wang, "Synthesis of magnetite/graphene oxide composite and application for cobalt(II) Removal," Journal of Physical Chemistry C, vol. 115, pp. 25234-25240, 2011.

[30] N. S. Chaudhari, A. P. Pandey, P. O. Patil, A. R. Tekade, S. B. Bari, and P. K. Deshmukh, "Graphene oxide based magnetic nanocomposites for efficient treatment of breast cancer," Materials Science and Engineering C, vol. 37, pp. 278-285, 2014.

[31] X. Yang, Y. Wang, X. Huang et al., "Multi-functionalized graphene oxide based anticancer drug-carrier with dualtargeting function and $\mathrm{pH}$-sensitivity," Journal of Materials Chemistry, vol. 21, pp. 3448-3454, 2011.

[32] A. R. K. Sasikala, R. G. Thomas, A. R. Unnithan et al., "Multifunctional nanocarpets for cancer theranostics: remotely controlled graphene nanoheaters for thermochemosensitisation and magnetic resonance imaging," Scientific Reports, vol. 6, no. 1, Article ID 20543, 2016.

[33] J. Li, S. Zhang, C. Chen et al., "Removal of $\mathrm{Cu}$ (II) and fulvic acid by graphene oxide nanosheets decorated with $\mathrm{Fe}_{3} \mathrm{O}_{4}$ nanoparticles," ACS Applied Materials \& Interfaces, vol. 4, no. 9, pp. 4991-5000, 2012.

[34] X. Yang, X. Zhang, Y. Ma et al., "Superparamagnetic graphene oxide- $\mathrm{Fe}_{3} \mathrm{O}_{4}$ nanoparticles hybrid for controlled targeted drug carriers," Journal of Materials Chemistry, vol. 19, pp. 2710-2714, 2009.

[35] F. He, J. Fan, D. Ma, L. Zhang, C. Leung, and H. L. Chan, "The attachment of $\mathrm{Fe}_{3} \mathrm{O}_{4}$ nanoparticles to graphene oxide by covalent bonding," Carbon, vol. 48, no. 11, pp. 3139-3144, 2010. 
[36] Y. Zhang, B. Chen, L. Zhang et al., "Controlled assembly of $\mathrm{Fe}_{3} \mathrm{O}_{4}$ magnetic nanoparticles on graphene oxide," Nanoscale, vol. 3, pp. 1446-1450, 2011.

[37] X. Fan, W. Peng, Y. Li et al., "Deoxygenation of exfoliated graphite oxide under alkaline conditions: a green route to graphene preparation," Advanced Materials, vol. 20, no. 23, pp. 4490-4493, 2008.

[38] T. Szabo, Z. Peter, E. Illes, L. Janovák, and A. Talyzin, "Stability and dye inclusion of graphene oxide/polyelectrolyte layer-by-layer self-assembled films in saline, acidic and basic aqueous solutions," Carbon, vol. 111, pp. 350-357, 2017.

[39] T. Yoon, J. Kim, J. Kim, J. Kim, and J. Lee, "Electrostatic selfassembly of $\mathrm{Fe}_{3} \mathrm{O}_{4}$ nanoparticles on graphene oxides for high capacity lithium-ion battery anodes," Energies, vol. 6, no. 9, pp. 4830-4840, 2013.

[40] T. Jiao, Y. Liu, Y. Wu et al., "Facile and scalable preparation of graphene oxide-based magnetic hybrids for fast and highly efficient removal of organic dyes," Scientific Reports, vol. 5, no. 1, Article ID 12451, 2015.

[41] M. Namvari and H. Namazi, "Clicking graphene oxide and $\mathrm{Fe}_{3} \mathrm{O}_{4}$ nanoparticles together: an efficient adsorbent to remove dyes from aqueous solutions," International Journal of Environmental Science and Technology, vol. 11, no. 6, pp. 1527-1536, 2014.

[42] A. R. M. Cornell and U. Schwertmann, The Iron Oxides, p. 573, Weinheim, Germany, VCH, 1996.

[43] E. Illés, E. Tombácz, M. Szekeres, I. Y. Tóth, Á. Szabó, and B. Iván, "Novel carboxylated PEG-coating on magnetite nanoparticles designed for biomedical applications," Journal of Magnetism and Magnetic Materials, vol. 380, pp. 132-139, 2015.

[44] E. Tombácz, E. Illés, A. Majzik, A. Hajdú, N. Rideg, and M. Szekeres, "Ageing in the inorganic nanoworld: example of magnetite nanoparticles in aqueous medium," Croatica Chemica Acta, vol. 80, pp. 503-515, 2007.

[45] D. Nesztor, K. Bali, I. Y. Tóth, M. Szekeres, and E. Tombácz, "Controlled clustering of carboxylated SPIONs through polyethylenimine," Journal of Magnetism and Magnetic Materials, vol. 380, pp. 144-149, 2015.

[46] I. Y. Tóth, D. Nesztor, L. Novák et al., "Clustering of carboxylated magnetite nanoparticles through polyethylenimine: covalent versus electrostatic approach," Journal of Magnetism and Magnetic Materials, vol. 427, pp. 280-288, 2017.

[47] F. Tuinstra and J. L. Koenig, "Raman Spectrum of Graphite," Journal of Chemical Physics, vol. 53, no. 3, pp. 1126-1130, 1970.

[48] A. Gupta, G. Chen, P. Joshi, S. Tadigadapa, and P. C. Eklund, "Raman scattering from high-frequency phonons in supported n-graphene layer films," Nano Letters, vol. 6, pp. 2667-2673, 2006.

[49] R. P. Vidano, D. B. Fischbach, L. J. Willis, and T. M. Loehr, "Observation of Raman band shifting with excitation wavelength for carbons and graphites," Solid State Communications, vol. 39, no. 2, pp. 341-344, 1981.

[50] A. C. Ferrari, "Raman spectroscopy of graphene and graphite: disorder, electron-phonon coupling, doping and nonadiabatic effects," Solid State Communications, vol. 143, no. 1-2, pp. 47-57, 2007.

[51] R. Saito, M. Hofmann, G. Dresselhaus, A. Jorio, and M. S. Dresselhaus, "Raman spectroscopy of graphene and carbon nanotubes," Advances in Physics, vol. 30, no. 3, pp. 413-550, 2011.
[52] S. Reich and C. Thomsen, "Raman spectroscopy of graphite," Philosophical Transactions of the Royal Society A, vol. 362, no. 1824, pp. 2271-2288, 2004.

[53] T. Szabó, O. Berkesi, P. Forgó et al., "Evolution of surface functional groups in a series of progressively oxidized graphite oxides," Chemistry of Materials, vol. 18, pp. 2740$2749,2006$.

[54] A.B. Bourlinos, A. Bakandritsos, V. Georgakilas, V. Tzitzios, and D. Petridis, "Facile synthesis of capped $\gamma-\mathrm{Fe}_{2} \mathrm{O}_{3}$ and $\mathrm{Fe}_{3} \mathrm{O}_{4}$ nanoparticles," Journal of Materials Science, vol. 41, no. 16 , pp. 5250-5256, 2006

[55] T. Szabó, V. Hornok, R. A. Schoonheydt, and I. Dékány, "Hybrid Langmuir-Blodgett monolayers of graphite oxide nanosheets," Carbon, vol. 48, no. 5, pp. 1676-1680, 2010.

[56] Y.-H. Lien and T.-M. Wu, "Preparation and characterization of thermosensitive polymers grafted onto silica-coated iron oxide nanoparticles," Journal of Colloid and Interface Science, vol. 326, no. 2, pp. 517-521, 2008.

[57] E. Tombacz, A. Hajdu, E. Illes, K. László, G. Garberoglio, and P. Jedlovszky, "Water in contact with magnetite nanoparticles, as seen from experiments and computer simulations," Langmuir, vol. 25, pp. 13007-13014, 2009.

[58] R. J. Hunter, R. H. Ottewill, and R. L. Rowell, Zeta Potential in Colloid Science, Principle and Application, p. 219, London, UK, Academic Press, 1981.

[59] R. L. D. Whitby, V. M. Gun'ko, A. Korobeinyk et al., "Driving forces of conformational changes in single-layer graphene oxide," ACS Nano, vol. 6, no. 5, pp. 3967-3973, 2012.

[60] L.-Z. Bai, D.-L. Zhao, Y. Xu et al., "Inductive heating property of graphene oxide- $\mathrm{Fe}_{3} \mathrm{O}_{4}$ nanoparticles hybrid in an $\mathrm{AC}$ magnetic field for localized hyperthermia," Materials Letters, vol. 68, pp. 399-401, 2012.

[61] D. Ortega and Q. A. Pankhurst, "Magnetic hyperthermia," Nanoscience, Royal Society of Chemistry, vol. 1, pp. 60-88, Royal Society of Chemistry, London, UK, 2013.

[62] M. Molcan, H. Gojzewski, A. Skumiel et al., "Energy losses in mechanically modified bacterial magnetosomes," Journal of Physics D: Applied Physics, vol. 49, Article ID 365002, 2016.

[63] G. Xie, P. Xi, H. Liu et al., "A facile chemical method to produce superparamagnetic graphene oxide- $\mathrm{Fe}_{3} \mathrm{O}_{4}$ hybrid composite and its application in the removal of dyes from aqueous solution," Journal of Materials Chemistry, vol. 22, no. 3, pp. 1033-1039, 2012.

[64] N. A. Travlou, G. Z. Kyzas, N. K. Lazaridis, and E. A. Deliyanni, "Functionalization of graphite oxide with magnetic chitosan for the preparation of a nanocomposite dye adsorbent," Langmuir, vol. 29, no. 5, pp. 1657-1668, 2013.

[65] Y. Dong, H. Zhang, Z. U. Rahman et al., "Graphene oxide- $\mathrm{Fe}_{3} \mathrm{O}_{4}$ magnetic nanocomposites with peroxidase-like activity for colorimetric detection of glucose," Nanoscale, vol. 4, no. 13, pp. 3969-3976, 2012.

[66] Y. Fu, J. Wang, Q. Liu, and H. Zeng, "Water-dispersible magnetic nanoparticle-graphene oxide composites for selenium removal," Carbon, vol. 77, pp. 710-721, 2014.

[67] L. Zhou, H. Deng, J. Wan, J. Shi, and T. Su, "A solvothermal method to produce $\mathrm{RGO}-\mathrm{Fe}_{3} \mathrm{O}_{4}$ hybrid composite for fast chromium removal from aqueous solution," Applied Surface Science, vol. 283, pp. 1024-1031, 2013. 


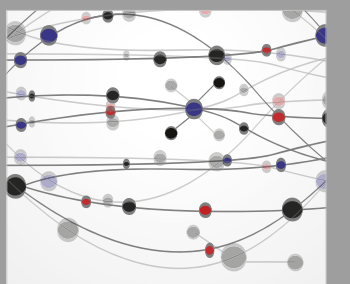

The Scientific World Journal
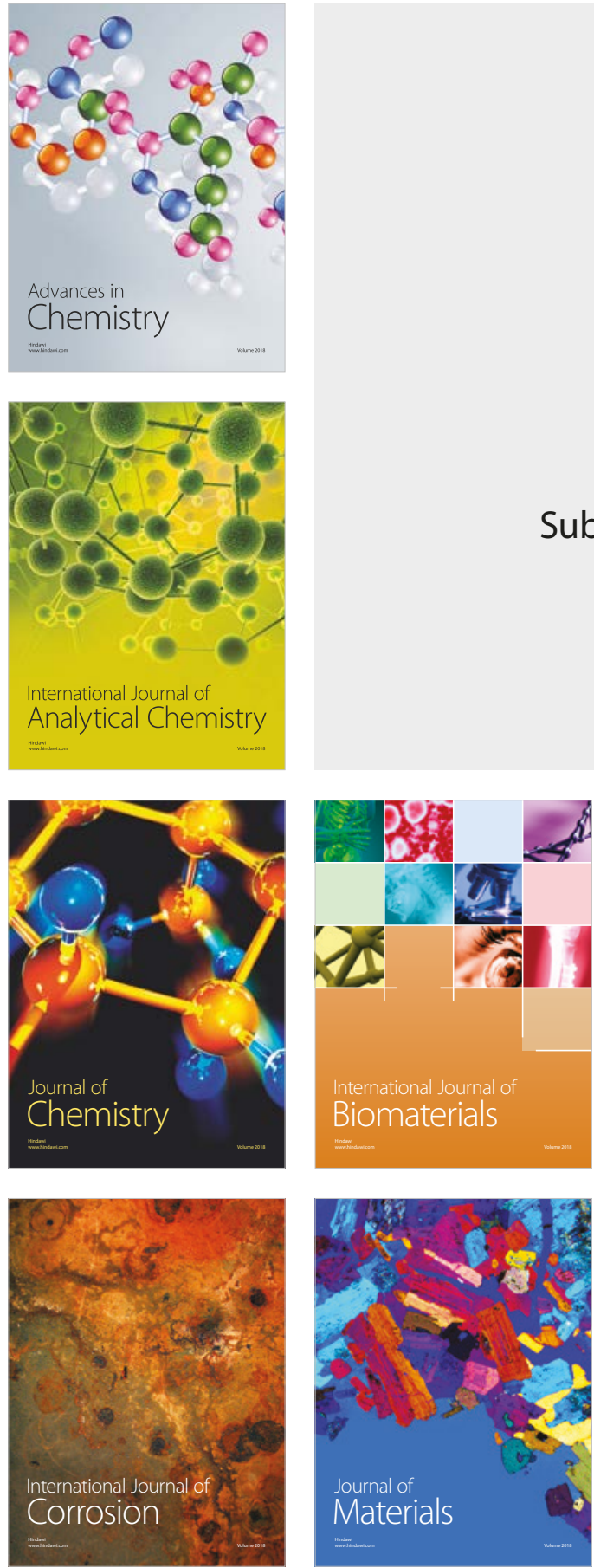

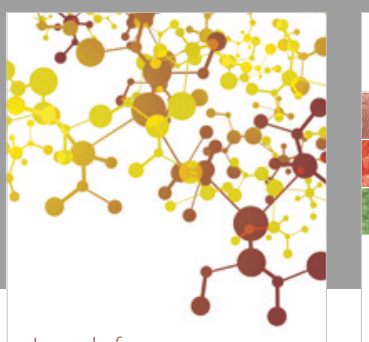

Journal of

Applied Chemistry
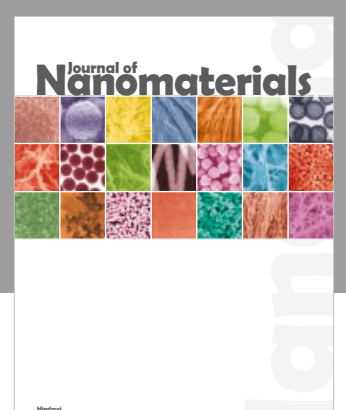

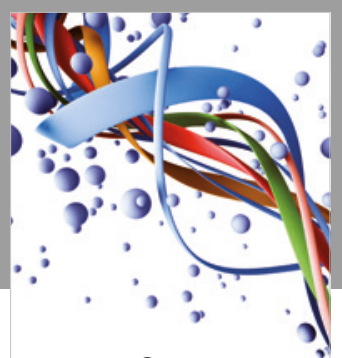

Scientifica

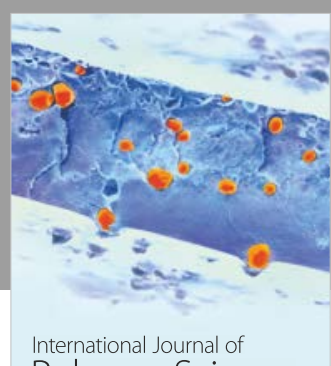

Polymer Science

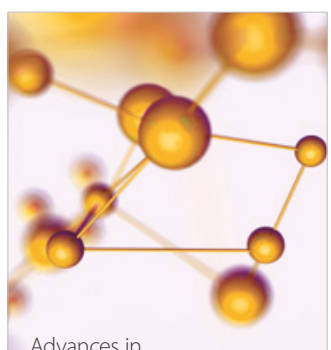

Physical Chemistry
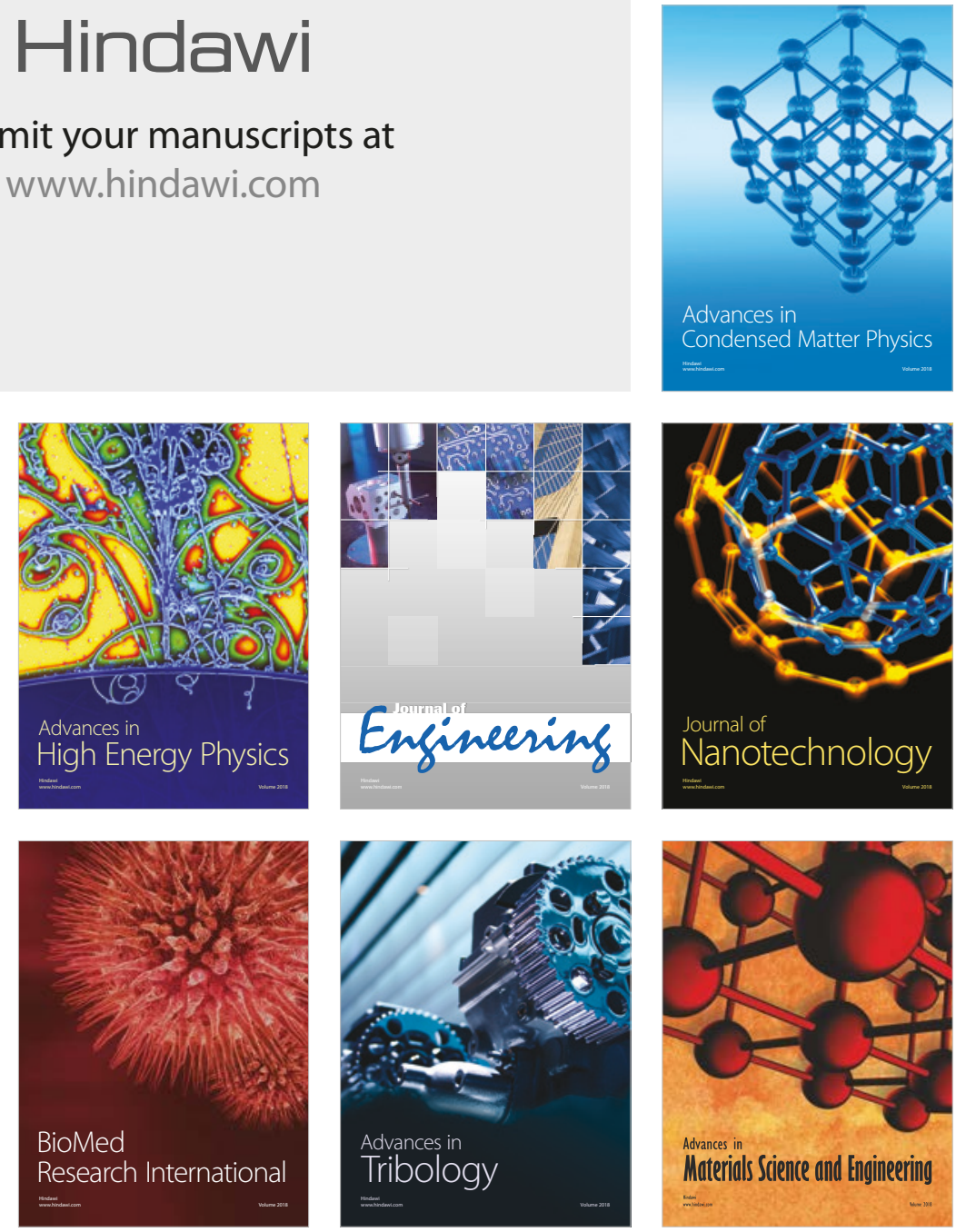